\title{
THREE-DIMENSIONAL DESINGULARIZED BOUNDARY INTEGRAL METHODS FOR POTENTIAL PROBLEMS
}

\author{
YUSONG CAO, WILLIAM W. SCHULTZ AND ROBERT F. BECK \\ University of Michigan, Ann Arbor, MI 48109, U.S.A.
}

\begin{abstract}
SUMMARY
The concept of desingularization in three-dimensional boundary integral computations is re-examined. The boundary integral equation is desingularized by moving the singular points away from the boundary and outside the problem domain. We show that the desingularization gives better solutions to several problems. As a result of desingularization, the surface integrals can be evaluated by simpler techniques, speeding up the computation. The effects of the desingularization distance on the solution and the condition of the resulting system of algebraic equations are studied for both direct and indirect versions of the boundary integral method. Computations show that a broad range of desingularization distances gives accurate solutions with significant savings in the computation time. The desingularization distance must be carefully linked to the mesh size to avoid problems with uniqueness and ill-conditioning. As an example, the desingularized indirect approach is tested on unsteady non-linear three-dimensional gravity waves generated by a moving submerged disturbance; minimal computational difficulties are encountered at the truncated boundary.
\end{abstract}

KEY wORDS Boundary integrals Potential problems Three-dimensional waves

\section{INTRODUCTION}

Boundary integral methods provide a powerful technique for the solution of linear, homogeneous boundary value problems. The method employs a fundamental solution, which satisfies the differential equation (and possibly part of the boundary conditions), to reformulate the problem as an integral equation on the boundary. In conventional boundary integral formulations, singularities of the fundamental solution are placed on the domain boundary. This requires special evaluation of singular integrands, which can result in costly numerical calculations. In time-dependent non-linear free surface problems ${ }^{1-3}$ a boundary integral problem is solved at each time step. Since most of the computation time is devoted to the boundary integral problem, an effective solution method is critical in the time-marching procedure.

When the singularity of the fundamental solution is placed away from the boundary and outside the domain of the problem, a desingularized boundary integral equation is obtained. We will show two advantages to this desingularization: a more accurate solution can be obtained for a given truncation, and a numerical quadrature can be used to reduce the computational time to obtain the algebraic system representing the discretized boundary integral problem. There are two types of non-singular boundary integral formulations: direct and indirect. In the direct method, Green's second identity is used to derive the boundary integral equation, and the solution of the problem is obtained directly by solving the boundary integral equation. In the 
indirect method, a boundary integral equation for the singularity strength is formulated where the singularity distribution is outside the problem domain. These two methods are formulated in the following section.

The first use of a desingularized method is the classical work by Von Kármán ${ }^{4}$ that determines the flow about axisymmetric bodies using an axial source distribution. The strength of the source distribution is determined by the kinematic boundary condition on the body surface. Kupradze ${ }^{5}$ proposes locating the boundary nodes on an auxiliary boundary outside the problem domain. Heise $^{6}$ studies some numerical properties of integral equations where the singular points are on an auxiliary boundary outside the problem domain for plane elastostatic problems. By applying Green's theorem to the solution and a simple wave source with the singular point lying inside the body (i.e. outside the problem domain) and using a bilinear expansion of the source, Martin ${ }^{7}$ obtains unique solutions of the so-called "null-field equations for the water wave radiation problems'. Han and Olson ${ }^{8}$ and Johnson and Fairweather ${ }^{9}$ use an adaptive method where singularities are located outside the domain and allowed to move as part of the solution process. This adaptive method requires considerably fewer singularities than the number of boundary nodes, but it results in a system of non-linear algebraic equations for both the strength and the location of the singularities. All these studies can be classified as non-singular methods and show a considerable reduction in the computation time.

The most complete discussion of a desingularization technique is given by Webster, ${ }^{10}$ who uses a triangular mesh of a singularity distribution (a simple source distribution) placed somewhat inside the surface of an arbitrary, three-dimensional smooth body to find the external potential. Analytical integration is used for each triangle using a linear distribution of singularity strength. These integrations require evaluation of (logarithmic and arctangent) transcendental functions. From the numerical results, Webster concludes that 'submergence of the singularity sheet below the surface of the body appears to improve greatly the accuracy, as long as the sheet is not submerged too far'. This suggests that for a certain discretization of the body surface one may obtain a more accurate solution by the non-singular formulation than by the singular formulation. Although Webster did not attempt it, a simple numerical quadrature can be used if the distance of desingularization is sufficiently large. This will significantly reduce the computational effort required to obtain the algebraic system representing the discretized boundary integral problem.

Although non-singular formulations of the indirect method have grown popular recently, few studies on the desingularized direct method have been reported, especially for three-dimensional problems. Schultz and Hong ${ }^{11}$ obtain a non-singular direct formulation by moving the singularity in the Cauchy integral away from the boundary in two-dimensional potential problems. Their results also show the advantages of the non-singular formulation. They also use an overdetermined system combining the real and imaginary parts of Cauchy's theorem or using 'extra' evaluation points away from the boundary contour. This overdetermined system can exhibit higher-order convergence than the determined system from the real or imaginary part of Cauchy's theorem.

Fewer investigations report on applications with boundaries extending to infinity, especially for wave problems. Jensen et al. ${ }^{12}$ solve the steady non-linear ship wave problem by the indirect method using a simple source distribution above a free surface, with minimal difficulties at the truncated boundary. While they use an upwinding technique as a form of the radiation condition, we find that the unsteady problem can be described by a technique that uses no special treatment for fixed time at the truncated boundary as long as a desingularized method is used.

After formulating the two desingularized methods in Section 2, we then examine the effect of the distance of desingularization on a simple problem with an infinite plane boundary. The 
convergence of the method with respect to mesh size and the computational time are compared to find 'optimum' desingularization distances. We then use this method to calculate the unsteady non-linear waves generated by a source-sink pair moving below a free surface.

\section{DESINGULARIZED BOUNDARY INTEGRAL METHOD}

The Laplace equation is the governing equation in a domain $D$ bounded by $\Gamma$. We assume that a Dirichlet condition is imposed on part of the boundary $\Gamma_{d}$ and a Neumann condition on the remaining boundary $\Gamma_{n}$ :

$$
\begin{aligned}
\Delta \phi & =0 & & \text { (in D), } \\
\phi & =\phi_{0} & & \left(\text { on } \Gamma_{\mathrm{d}}\right), \\
\partial \phi / \partial n & =\chi & & \text { (on } \left.\Gamma_{\mathrm{n}}\right),
\end{aligned}
$$

where $\phi_{0}$ and $\chi$ are known functions and $\partial / \partial n$ is the outward normal to the boundary $\Gamma_{\mathbf{d}}$. If the boundary extends to infinity, a far-field condition is required.

The desingularized boundary integral method separates the integration and control (i.e. evaluation) surfaces, one of which is the boundary of the problem. In the direct method the boundary of the problem is the integration surface, while in the indirect method the boundary is the control surface.

\subsection{Direct method}

The boundary integral equation in the direct method is derived from Green's second identity:

$$
\iiint_{D}(\phi \Delta \psi-\psi \Delta \phi) \mathrm{d} D-\iint_{\Gamma}\left(\phi \frac{\partial \psi}{\partial n}-\psi \frac{\partial \phi}{\partial n}\right) \mathrm{d} \Gamma=0,
$$

which holds for any two functions with second derivatives continuous in $D$ and the boundary $\Gamma$. If $\phi$ is the solution and $\psi$ is chosen as

$$
\psi=\frac{1}{\left|\mathbf{x}_{\mathbf{p}}-\mathbf{x}_{\mathbf{q}}\right|},
$$

with its singular points $\mathbf{x}_{\mathrm{p}}$ outside $D$ and $\mathbf{x}_{\mathrm{q}}$ on $\Gamma$, the volume integral in (4) becomes zero. We then have

$$
\iint_{\Gamma}\left(\phi \frac{\partial \psi}{\partial n}-\psi \frac{\partial \phi}{\partial n}\right) \mathrm{d} \Gamma=0
$$

Applying the boundary conditions (2) and (3) to (6) and moving the known quantities to the right-hand side gives

$$
\begin{aligned}
\iint_{\Gamma_{\mathbf{n}}} \phi\left(\mathbf{x}_{\mathbf{q}}\right) \frac{\partial}{\partial n}\left(\frac{1}{\left|\mathbf{x}_{\mathbf{p}}-\mathbf{x}_{\mathbf{q}}\right|}\right) \mathrm{d} \Gamma-\iint_{\Gamma_{\mathbf{d}}} \frac{1}{\left|\mathbf{x}_{\mathbf{p}}-\mathbf{x}_{\mathbf{q}}\right|} \frac{\partial \phi\left(\mathbf{x}_{\mathbf{q}}\right)}{\partial n} \mathrm{~d} \Gamma= \\
\quad-\iint_{\Gamma_{\mathbf{d}}} \phi_{0} \frac{\partial}{\partial n}\left(\frac{1}{\left|\mathbf{x}_{\mathbf{p}}-\mathbf{x}_{\mathbf{q}}\right|}\right) \mathrm{d} \Gamma+\iint_{\Gamma_{n}} \frac{1}{\left|\mathbf{x}_{\mathbf{p}}-\mathbf{x}_{\mathbf{q}}\right|} \chi \mathrm{d} \Gamma .
\end{aligned}
$$

The kernels are non-singular since $x_{p}$ and $x_{q}$ never coincide. The integral equation (7) is solved for $\phi$ on $\Gamma_{\mathrm{n}}$ and $\partial \phi / \partial n$ on $\Gamma_{\mathrm{d}}$. Then the solution in the problem domain $D$ can be expressed in terms 
of $\phi$ and $\partial \phi / \partial n$ on the boundaries:

$$
\begin{aligned}
4 \pi \phi(\mathbf{x})= & \iint_{\Gamma_{\mathbf{n}}} \phi\left(\mathbf{x}_{\mathbf{q}}\right) \frac{\partial}{\partial n}\left(\frac{1}{\left|\mathbf{x}-\mathbf{x}_{\mathbf{q}}\right|}\right) \mathrm{d} \Gamma-\iint_{\Gamma_{\mathbf{d}}} \frac{1}{\left|\mathbf{x}-\mathbf{x}_{\mathbf{q}}\right|} \frac{\partial \phi\left(\mathbf{x}_{\mathbf{q}}\right)}{\partial n} \mathrm{~d} \Gamma+\iint_{\Gamma_{\mathbf{d}}} \phi_{0} \frac{\partial}{\partial n}\left(\frac{1}{\left|\mathbf{x}-\mathbf{x}_{\mathbf{q}}\right|}\right) \mathrm{d} \Gamma \\
& -\iint_{\Gamma_{\mathbf{n}}} \frac{1}{\left|\mathbf{x}-\mathbf{x}_{\mathbf{q}}\right|} \chi \mathrm{d} \Gamma \quad(\mathbf{x} \in D) .
\end{aligned}
$$

\subsection{Indirect method}

The indirect method forms a solution by integrating a simple source distribution over some surface $\Omega$ outside the problem domain:

$$
\phi(\mathbf{x})=\iint_{\Omega} \sigma\left(\mathbf{x}_{\mathrm{s}}\right) \frac{1}{\left|\mathbf{x}-\mathbf{x}_{\mathrm{s}}\right|} \mathrm{d} \Omega .
$$

By applying the boundary conditions (2) and (3), we obtain boundary integral equations for the unknown strength of the singularities, $\sigma\left(\mathbf{x}_{\mathbf{s}}\right)$ :

$$
\begin{aligned}
\iint_{\Omega} \sigma\left(\mathbf{x}_{\mathrm{s}}\right) \frac{1}{\left|\mathbf{x}_{\mathrm{c}}-\mathbf{x}_{\mathrm{s}}\right|} \mathrm{d} \Omega & \left.=\phi_{0}\left(\mathbf{x}_{\mathrm{c}}\right) \quad \text { (on } \Gamma_{\mathrm{d}}\right), \\
\iint_{\Omega} \sigma\left(\mathbf{x}_{\mathrm{s}}\right) \frac{\partial}{\partial n}\left(\frac{1}{\left|\mathbf{x}_{\mathrm{c}}-\mathbf{x}_{\mathrm{s}}\right|}\right) \mathrm{d} \Omega & \left.=\chi\left(\mathbf{x}_{\mathrm{c}}\right) \quad \text { (on } \Gamma_{\mathfrak{n}}\right),
\end{aligned}
$$

where $\mathbf{x}_{\mathrm{s}}$ is the integration point on the surface $\Omega$ outside $D, \mathbf{x}_{\mathrm{c}}$ is the control point on $\Gamma$ and again $\partial / \partial n$ represents the derivative normal to $\Gamma$. Once the singularity strength is determined, the solution for $\phi$ can be obtained from (9). The term $1 /\left|\mathbf{x}_{\mathbf{c}}-\mathbf{x}_{\mathbf{s}}\right|$ can easily be replaced by other higher-order singularities (dipoles, etc.) with little additional computational effort since the integrals are non-singular and are evaluated numerically.

\subsection{Difficulties introduced by desingularization}

Desingularization results in a Fredholm integral equation of the first kind. This can lead to uniqueness and completeness problems of the solution as manifested in the ill-conditioning of the resulting algebraic system if the desingularization distance is not properly chosen. Uniqueness can be a serious problem for the direct method since the solution of the algebraic system is $\phi$. However, we can accept multiple constructions (different values of $\sigma$ ) of the same $\phi$ with the indirect method. Webster ${ }^{10}$ shows that the non-singular formulations are not significantly less well conditioned than singular formulations and that completeness is assured if the singularities of the numerical method are placed closer to the surface. He also discusses strategies for choosing the desingularization distance for closed bodies. The method we describe here uses a desingularization distance that is related to the local mesh size (described in more detail in Section 3). As the meshes become finer, the singular points approach the boundary. In the limit the non-singular formulation is consistent with the singular formulation. For example, the desingularized kernel (although never singular) can be shown to have pointwise convergence with the singular kernel everywhere except at the singular point as $l_{\mathrm{d}} \rightarrow 0$. The numerical integration error will converge if the singularity approaches the boundary at a sufficiently slow rate as the mesh is refined (as shown in Section 3). Since the singular indirect and direct methods are equivalent in the limit of infinite mesh refinement, ${ }^{13}$ the properties of the singular boundary integral equations still apply for both methods. 
Desingularization increases the condition number of the resulting linear system. In most three-dimensional computations the large number of unknowns requires an iterative solution of the linear system. As the condition number increases, one can expect a loss in accuracy or an increase in the number of iterations. Then there appears to be an 'optimum' desingularization distance. If the singularity is too far from the boundary, the linear system will be poorly conditioned and uniqueness and completeness problems occur. If the singularity is too close to the boundary, numerical integration of the singular terms is suspect and the solution may not be as accurate, even if 'exact' integration is used. Unfortunately, little guidance in the selection of the desingularization is available except for Webster's ${ }^{10}$ discussion for axisymmetric bodies. If the solution is sensitive to the desingularization distance, the non-singular formulation would not be very practical. We will show that this is not the case in the following examples.

\section{EXAMPLES}

First we test the numerical performance of the desingularized boundary integral methods on a problem where the potential is generated by a dipole below an infinite flat plane with $\phi=0$. We solve for $\partial \phi / \partial n$ on the plane. This simple problem has an exact solution formed by the dipole and its image about the flat plane. This problem represents the solution to the first time step of non-linear waves caused by an impulsive disturbance of a dipole under water. We then apply the method to time-dependent non-linear waves caused by an underwater disturbance (a moving source-sink pair in our calculation).

The direct and indirect methods are compared for the simpler example. In the direct method the boundary is divided into rectangles and a solution is sought at the nodes. Since the kernels are non-singular, the integrals can be evaluated using Gaussian quadrature, where the solution is assumed to be bilinear over each rectangle. In the indirect method the integrals of the singularity distribution (10) and (11) may be replaced by a summation of concentrated singularities without an apparent loss of accuracy when the desingularization distance is appropriate. This does not require integration and mapping in numerical calculations. Therefore the computations will be less complex and time-consuming than in the direct method.

A collocation method is used to solve both formulations of the boundary integral equations. In the direct method we satisfy Green's theorem at chosen points outside the problem domain, while in the indirect method we satisfy the boundary conditions at the collocation points on the boundary. We propose that the distance of a singular point from the boundary be given by

$$
L_{\mathrm{d}}=l_{\mathrm{d}}\left(D_{\mathrm{m}}\right)^{\alpha},
$$

where $l_{\mathrm{d}}$ is a parameter that reflects how far the integral equation is desingularized, $D_{\mathrm{m}}$ is the local mesh size (here we choose the square root of the local mesh area) and $\alpha$ is a parameter that must be chosen carefully.

To test the convergence with respect to mesh refinement, we evaluate the potential due to a known constant normal dipole distribution within a square, flat surface

$$
(-1<x<1,-1<y<1, z=0)
$$

at a point with a distance given by (12) above the centre of the surface. The surface is subdivided into a square mesh and $2 \times 2$ Gaussian quadrature is used. A third-order convergence should be found assuming the integrand is non-singular and is independent of the mesh. Although moving the singular points makes the integrand non-singular, (12) makes the integrand depend on the mesh size and hence third-order convergence is not assured. Figure 1 shows the convergence of the numerical integration for three values of $\alpha: 0,0.5$ and 1 . As long as $\alpha<1$, third-order 


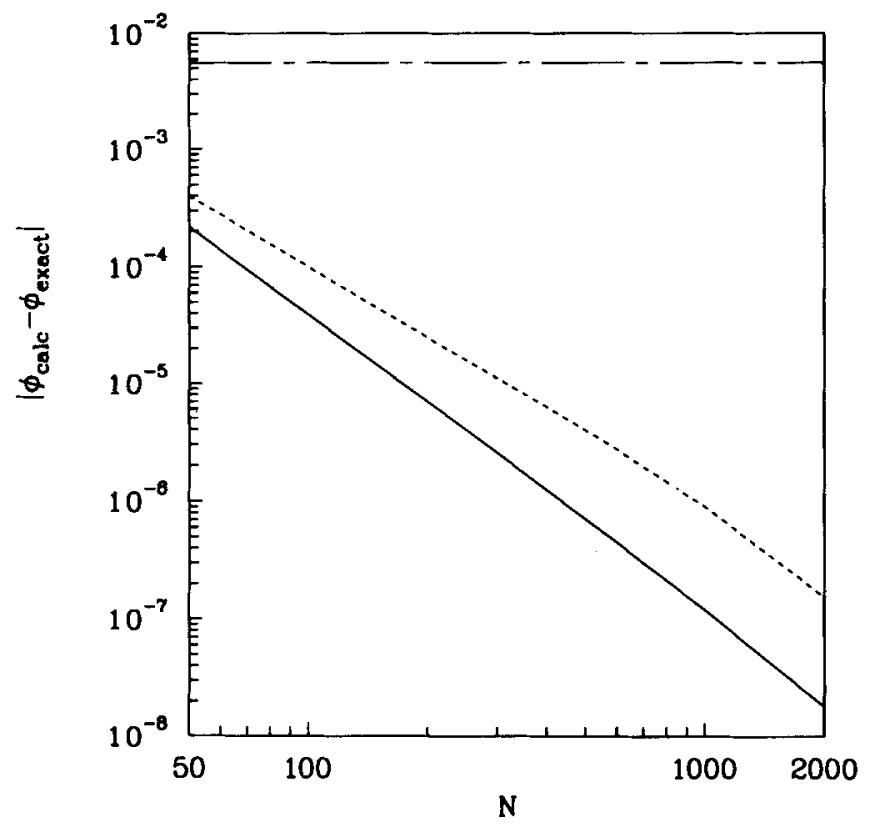

Figure 1. Convergence of the numerical integration: - - -,$\alpha=1 ;----, \alpha=0 \cdot 5 ;-\_, \alpha=0$

convergence is recovered, although the numerical integration error increases with $\alpha$. On the other hand, $\alpha$ should be greater than zero for the uniqueness and completeness properties of the solution of the integral equation. Therefore an appropriate $\alpha$ lies between zero and unity. In the following calculations we choose a value of $\alpha=0.5$. We then test the algorithm for a range of $l_{\mathrm{d}}$.

In the results that follow, a generalized minimal residual method (GMRES) ${ }^{14}$ iteratively solves the system of equations. This method iteratively minimizes the residual norm of a general $N \times N$ algebraic system as in a standard conjugate gradient routine for non-symmetric matrices, except with explicit orthogonalization of the search direction using a modified Gram-Schmidt procedure. For the examples with smaller degrees of freedom presented here, we have found this method to be approximately twice as fast as a least-squares conjugate gradient routine. A sufficiently small value of the convergence tolerance is chosen so that it introduces negligible error in comparison to that introduced by truncation. The computations are performed on an Apollo DN10000 workstation using 16-digit arithmetic.

\subsection{A dipole below a $\phi=0$ infinite flat plane}

In this example a Dirichlet condition, $\phi=0$, is imposed on the $z=0$ plane. A dipole of unit strength is located at $\mathbf{x}_{0}=(0,0,-1)$ and the direction of the dipole coincides with the $x$-axis. The normal derivative $\partial \phi / \partial n$ is sought on $z=0$.

Since the potential at the dipole location is singular for this problem, we decompose the solution into a regular and singular part: $\phi=\tilde{\phi}-(1 / 4 \pi) x_{\mathrm{p}} /\left|\mathbf{x}_{\mathrm{p}}-\mathbf{x}_{\mathbf{0}}\right|^{3}$. We then apply (7) for $\tilde{\phi}$ and recognize that $\Gamma_{n}$ is absent and the far field does not contribute. Substituting back for $\phi$ gives

$$
\iint_{\Gamma_{d}} \frac{\partial \phi}{\partial n} \frac{1}{\left|\mathbf{x}_{p}-\mathbf{x}_{q}\right|} \mathrm{d} \Gamma_{1}=\frac{x_{p}}{\left|\mathbf{x}_{p}-\mathbf{x}_{0}\right|^{3}},
$$


where $\mathbf{x}_{\mathrm{p}}$ is the control point outside $D$ and $\mathbf{x}_{\mathrm{p}}$ is the integration point on $\Gamma_{\mathrm{d}}$. In our computation the computational domain is discretized into a uniform square mesh defined by $N$ node points after truncating the $z=0$ plane $\left(\Gamma_{\mathrm{d}}\right)$ at $x= \pm R_{\infty}$ and $y=R_{\infty}$ with a symmetry condition on $y=0$. The integrations assume a bilinear distribution of $\partial \phi / \partial n$ and use a $2 \times 2$ Gaussian quadrature. The control points are placed directly above the nodal points at a distance $L_{\mathrm{d}}=l_{\mathrm{d}}(\Delta x)^{1 / 2}$, where $\Delta x$ is the mesh spacing.

In the indirect method we construct the potential using (9), with the integration replaced by summation as discussed previously, supplemented with the singular dipole term

$$
\phi(\mathbf{x})=\frac{-1}{4 \pi} \frac{x}{\left|\mathbf{x}-\mathbf{x}_{0}\right|^{3 / 2}}+\sum_{i=1}^{N} \frac{\sigma_{i}}{\left|\mathbf{x}-\mathbf{x}_{\mathrm{s} i}\right|},
$$

where $\mathbf{x}_{\mathrm{s} i}$ are the source points above $z=0$ and $\sigma_{i}$ are constants to be determined. The first term is the contribution from the dipole and the rest from the $z=0$ plane. The boundary condtion $\phi=0$ on $z=0$ results in

$$
\sum_{i=1}^{N} \frac{\sigma_{i}}{\left|\mathbf{x}_{\mathrm{c}}-\mathbf{x}_{\mathrm{s} i}\right|}=\frac{1}{4 \pi} \frac{x_{\mathrm{c}}}{\left[x_{\mathrm{c}}^{2}+y_{\mathrm{c}}^{2}+\left(z_{\mathrm{c}}+1\right)^{2}\right]^{3 / 2}},
$$

where $\mathbf{x}_{\mathrm{c}}=\left(x_{\mathrm{c}}, y_{\mathrm{c}}, z_{\mathrm{c}}\right)$ is the control point on $z=0$.

The exact solution is

$$
\phi(x, y, z)=-\frac{-1}{4 \pi} \frac{x}{\left[x^{2}+y^{2}+(z+1)^{2}\right]^{3 / 2}}+\frac{1}{4 \pi} \frac{x}{\left[x^{2}+y^{2}+(z-1)^{2}\right]^{3 / 2}} .
$$

The exact solution for the normal velocity on the $z=0$ plane then becomes

$$
\left(\frac{\partial \phi}{\partial n}\right)_{\text {exact }}=\frac{3}{2 \pi} \frac{x}{\left(x^{2}+y^{2}+1\right)^{5 / 2}} .
$$

The results of the normal velocity are compared to (17) by the RMS error, defined by

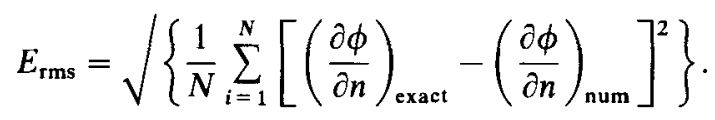

The mesh configuration is shown in Figure 2.

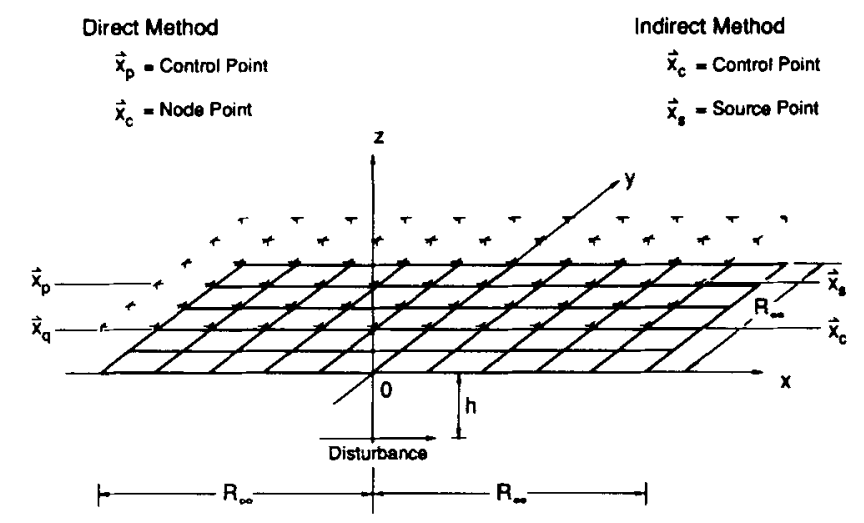

Figure 2. Schematic diagram of a dipole below a $\phi=0$ infinite flat plane 
Figure 3 shows the RMS errors for this example using the direct and indirect methods for three different values of $N$ while varying the desingularization distance. The direct method using $2 \times 2$ Gaussian quadrature shows a rapid drop in error near zero desingularization followed by increasing error as $l_{\mathrm{d}}$ increases. In the indirect method (using isolated sources $\mathbf{x}_{\mathrm{s} i}$ right above the control points $\mathbf{x}_{\mathrm{c}}$ ) the solution blows up for $l_{\mathrm{d}}=0$ because the integration and control points coincide. However, as $l_{\mathrm{d}}$ increases, the errors decrease rapidly and the solutions are rather insensitive to the variation of $l_{\mathrm{d}}$ for quite a large range. Eventually, $l_{\mathrm{d}}$ becomes too large for the given truncation to represent the second term in (16) well and the errors start to increase.

We have performed some limited computations where the control and nodal points are staggered. These staggered computations give similar results for optimal desingularization but give significantly better results than the non-staggered algorithm only when the desingularization is very small. Since staggering strategies are difficult to choose and only marginal improvement is obtained, we avoid the staggered method. We also tried using a surface distribution of sources above the $z=0$ plane in the indirect method for this problem. This is similar to Webster's method $^{10}$ except that the integrals over the source surface are performed using $2 \times 2$ Gaussian quadrature. As seen in Figure 4, the results are better than those of isolated sources for a large range of $l_{\mathrm{d}}$ (from 0.1 to 3.0 ). This can be expected since the use of isolated sources implies using a rougher quadrature for the integrals over the source surface. However, we found that the condition number of the resulting system of equations using a surface distribution of sources is greater than that using isolated by an order of 100 . Since it does not improve the optimum accuracy, it takes considerably longer to form the algebraic system, and because the iterative solution will take longer for the distributed source, we find that non-staggered, isolated sources are preferred.

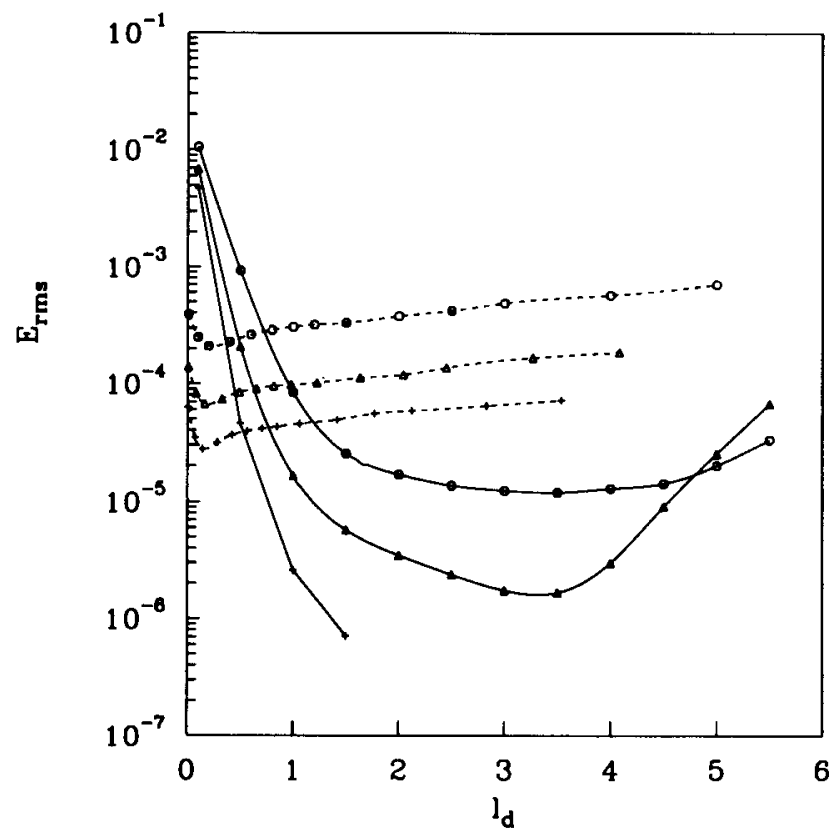

Figure 3. Effects of desingularization on error $\left(R_{\infty}=6.667\right):----$, direct method; - , indirect method; $O, N=231$; $\triangle, N=496 ;+, N=861$ 


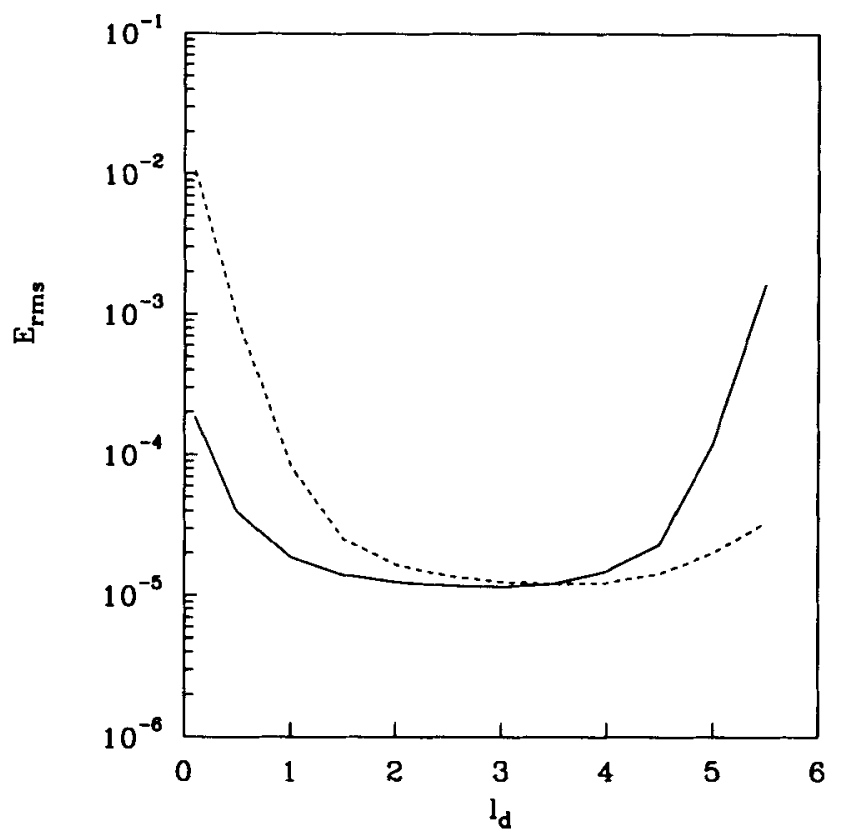

Figure 4. Comparison of surface distribution and isolated sources for indirect method $\left(R_{\infty}=6.667, N=231\right)$ : $--\infty$, isolated source; - -

A comparison of different techniques used to evaluate the influence function for the direct method is given in Figure 5. As expected for small $l_{\mathrm{d}}$, the error using the Gaussian quadrature is larger than the error using analytic integration ${ }^{15}$ because the integration error dominates the accuracy of the solution. As $l_{\mathrm{d}}$ increases, the Gaussian quadrature integrates the smoother integrands accurately and the results merge with those for analytic integration for all $l_{\mathrm{d}}$. It is fortuitous that in the middle range of $l_{\mathrm{d}}$ the results of the Gaussian quadrature show more accuracy than those using analytic integration--the discretization and numerical quadrature errors apparently tend to cancel each other. A more accurate $3 \times 3$ Gaussian quadrature brings the results closer to those from analytic integration, as expected. Although not shown here, increasing the number of nodes, $N$, also brings the results of the Gaussian and exact quadrature closer together.

Desingularization in the indirect method greatly reduces the error as can be seen in Figures 3 and 4. Webster ${ }^{10}$ came to the same conclusion using analytic integration with a distributed source. Desingularization is apparently more beneficial in the indirect method because the solution of many problems can be represented accurately by a finite number of some singularities located outside the problem domain. In this example, one negative image dipole above the $z=0$ plane results in the exact solution. Desingularization may be more problem (or geometry) specific for the indirect method.

The effect of desingularization on the condition number of the system of linear equations is shown in Figure 6 for the direct and indirect methods. The condition number increases exponentially with $l_{\mathrm{d}}$. However, a poorly conditioned system does not necessarily imply an inaccurate solution. Minimal errors occur around $l_{\mathrm{d}}=3.0$ in the indirect method, where the condition number is quite large. The increased condition number is likely to increase the number of 


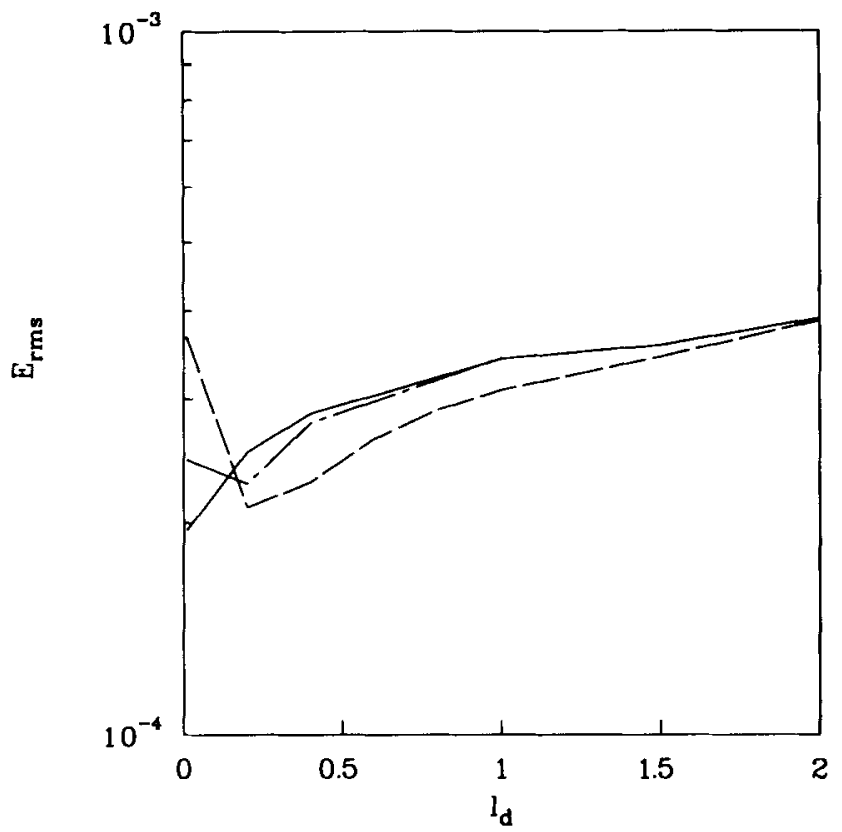

Figure 5. Comparison between analytic and Gaussian quadrature $\left(R_{\infty}=6.667, N=231\right) ;-\ldots$, analytic; - - _ $2 \times 2$ Gaussian quadrature; - - -, $3 \times 3$ Gaussian quadrature

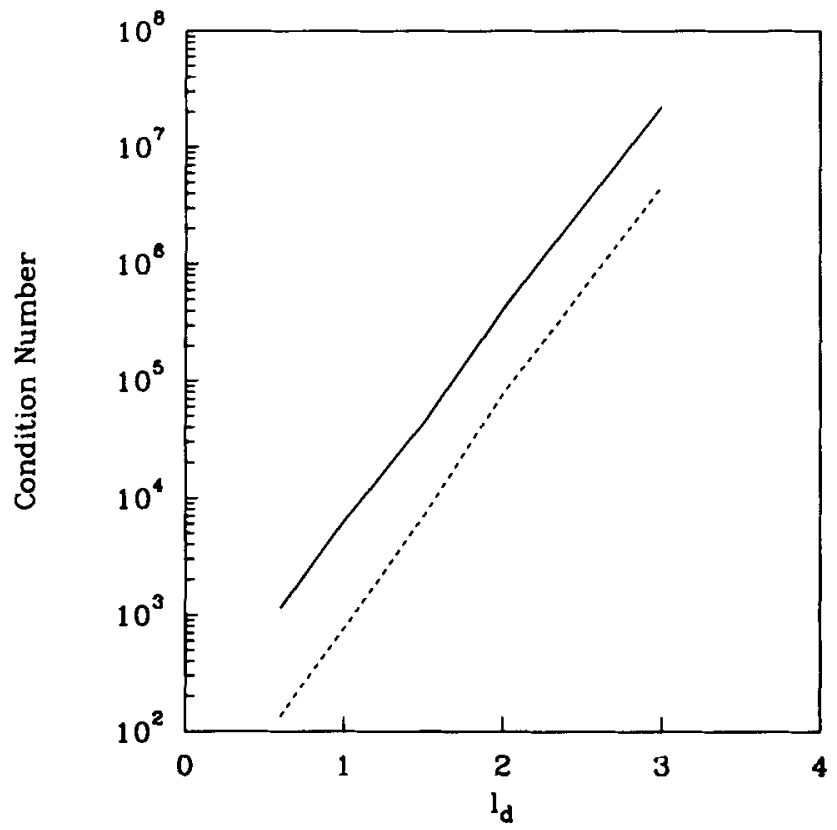

Figure 6. Effect of desingularization on condition number $\left(R_{\infty}=6.667, N=231\right):--$, direct method; -----, indirect method 
iterations and the solution should be tested for precision dependence (no significant dependence was found here). Figure 7 compares the ratios of CPU time by GMRES to that by an LU algorithm for the solution as a function of $l_{\mathrm{d}}$ for $N=231$. The LU algorithm takes about $3.5 \mathrm{~s}$ to solve the system. An error tolerance $\varepsilon$ for the least-squares residual of the equations needs to be specified when GMRES is used. We find that $\varepsilon=10^{-5}$ is sufficiently small in all the computations presented here. With the use of this tolerance, the CPU time by GMRES is less than that by the LU algorithm for $l_{\mathrm{d}}<1.5$. Analytic integration with a bilinear source distribution ${ }^{15}$ requires about $22 \mathrm{~s}$ to set up the matrix while the $2 \times 2$ Gaussian quadrature requires only about $6.4 \mathrm{~s}$, for a saving of about $70 \%$. However, the CPU time for solving the system varies from 3.0 to $4.0 \mathrm{~s}$ as $l_{\mathrm{d}}$ changes from 0.8 and 3.0 . Even in the worst case, $l_{\mathrm{d}}=3.0$, we gain a total reduction in CPU time of about $60 \%$ over the singular formulation $\left(l_{\mathrm{d}}=0\right)$ using analytic integration. In the indirect method the CPU time for the matrix set-up is only about $1.3 \mathrm{~s}$ and the CPU for the solution of the system varies from 1.7 to $4.0 \mathrm{~s}$. The total CPU time is reduced by about $80 \%$ with the indirect method for this truncation. When the desingularization distance is small (e.g. $l_{\mathrm{d}}<1 \cdot 5$ ), a larger $\varepsilon$ does not result in a significant difference in RMS error; see Figure 8. However, the CPU time is significantly reduced; see Figure 9.

Figure 10 shows the effect of truncating the infinite plane. The computational domain is extended by adding uniformly sized meshes. Both the direct and indirect methods converge quadratically with respect to the extent of the computational domain for this problem, as expected. Both methods also converge algebraically (approximately linearly) with respect to $N$, as can be seen in Figure 11, where the computational domain remains unchanged while the mesh size varies. The mesh convergence is algebraic for all $l_{\mathrm{d}}$ (not shown here).

Figure 12 shows the error distribution along the $x$-axis for both methods. Larger oscillations in the solution by the direct method are observed at the edge of the computational domain. This

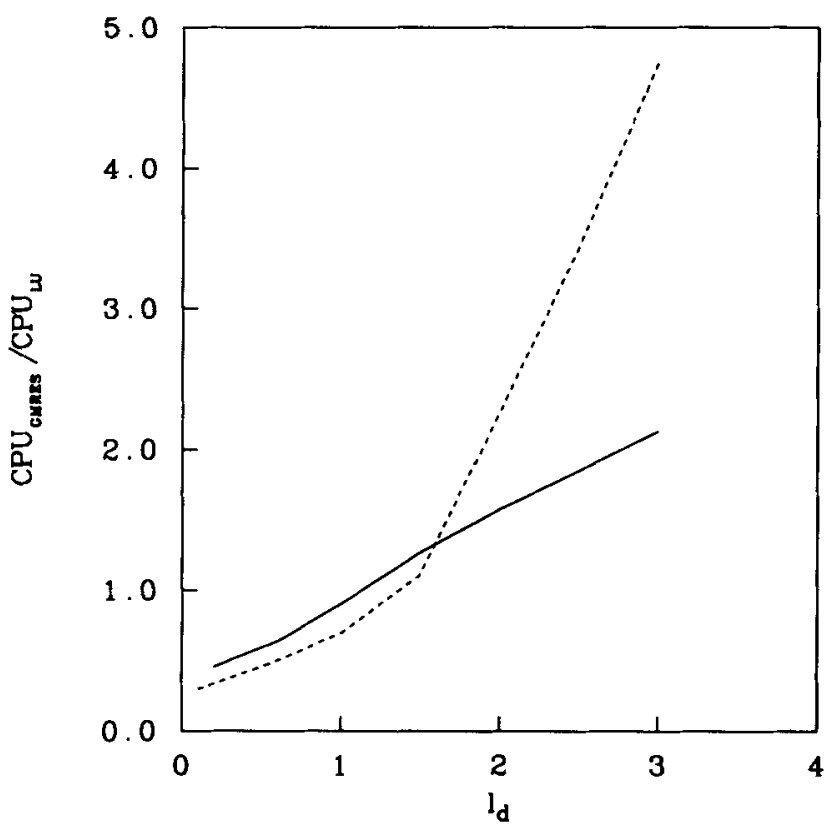

Figure 7. Effect of desingularization on computational time $\left(R_{\infty}=6.667, N=231\right)$ : - - , direct method; - ---, indirect method 


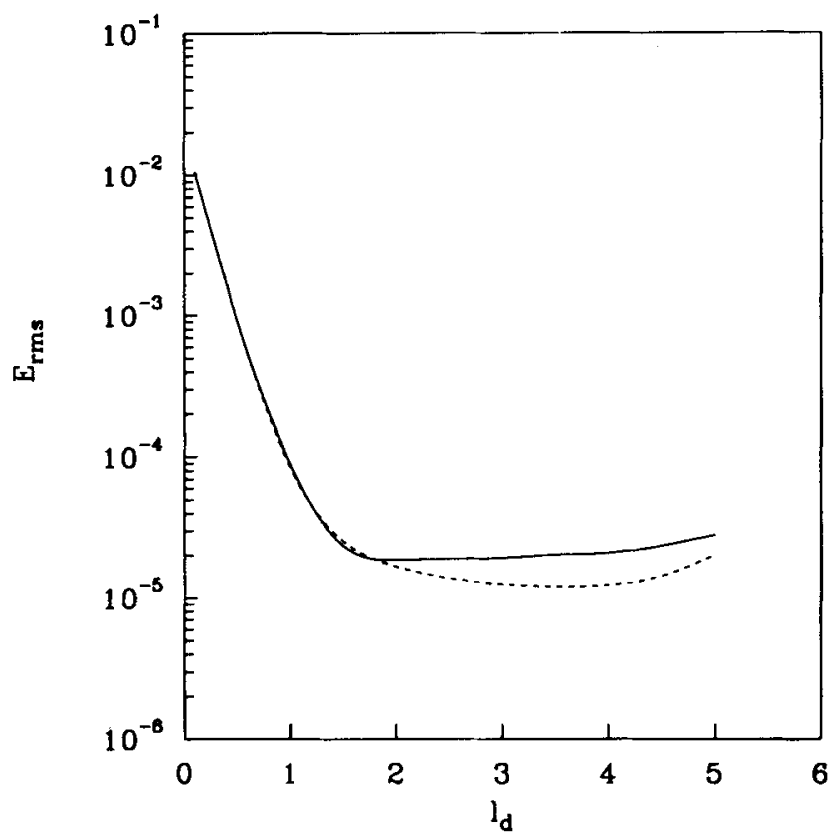

Figure 8. Effect of iterative tolerance on error $\left(R_{\infty}=6 \cdot 667, N=231\right.$; indirect method $)$ : -,$\varepsilon=10^{-2}$; ----, $\varepsilon=10^{-s}$

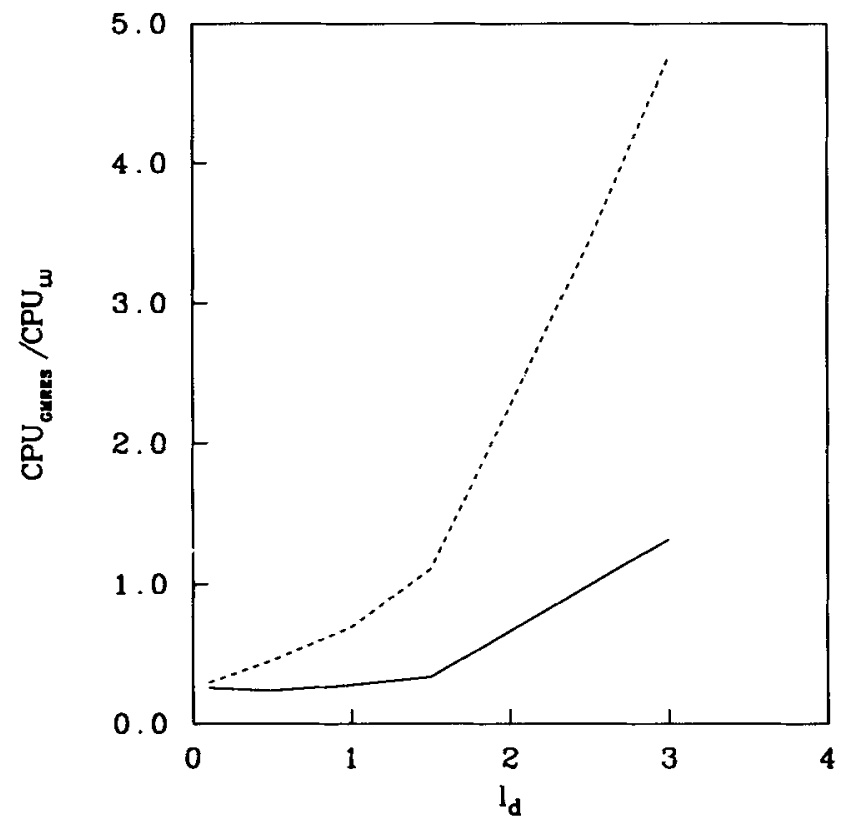

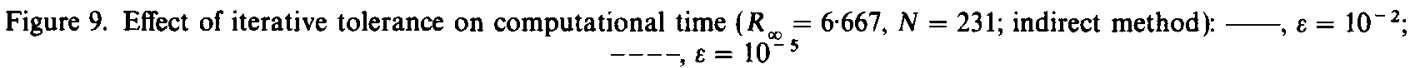




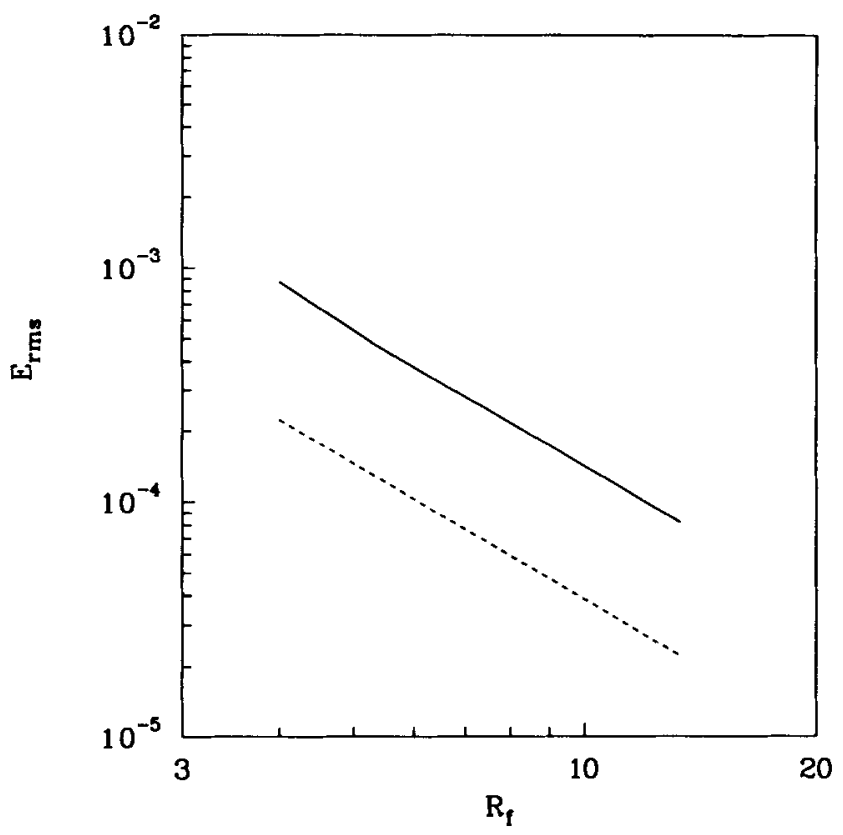

Figure 10. Convergence with respect to truncation of boundary at infinity $\left(\Delta X=0.6667, l_{\mathrm{d}}=1\right)$ : - , direct method; $-\ldots$, indirect method

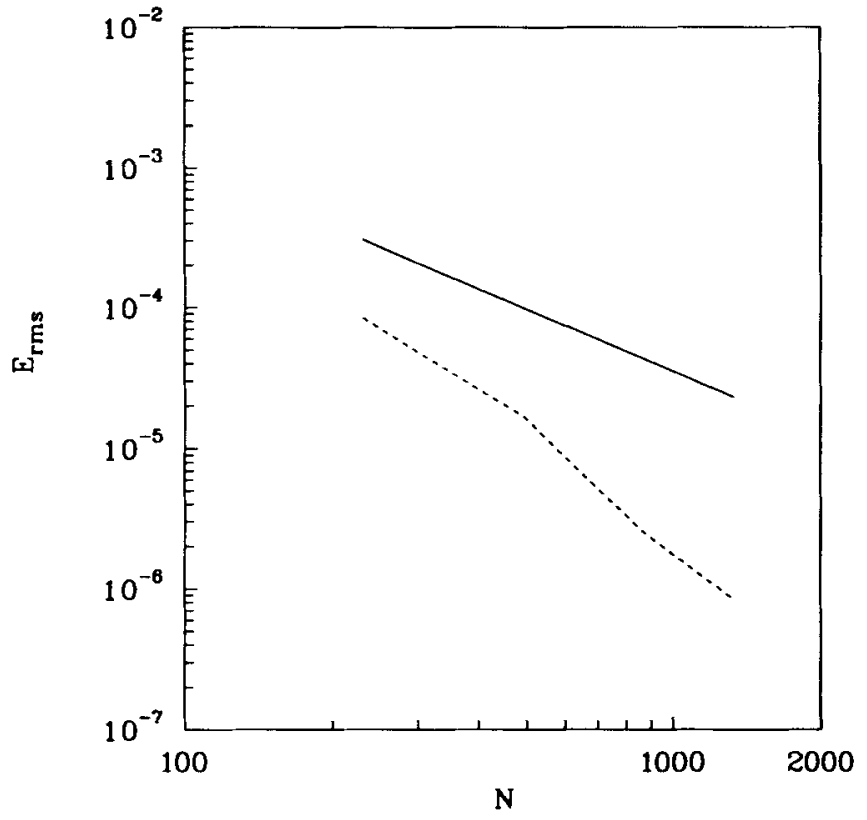

Figure 11. Convergence with respect to number of nodes $\left(R_{\infty}=6.667, l_{\mathrm{d}}=1 \cdot 0\right):-$, direct method, ---- , indirect 


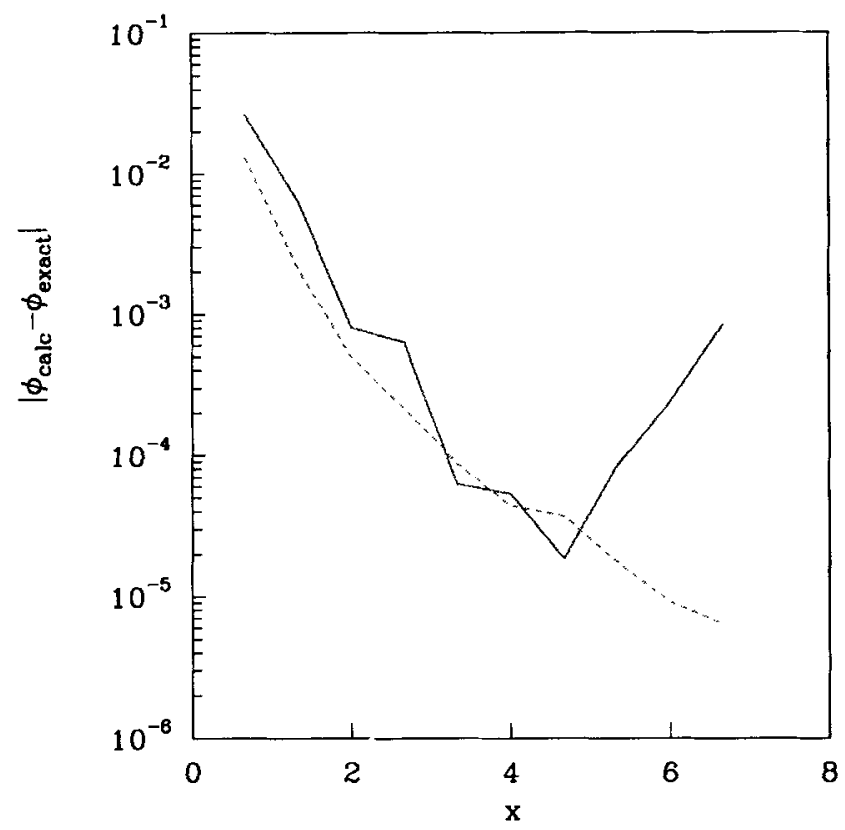

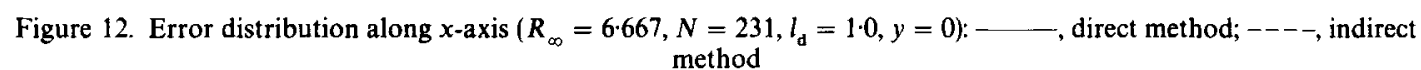

may be due to the neglect of the contribution from the integrals over the far-field closure in the direct method, which very likely results in a strong global effect on the accuracy of the boundary integral equation itself. In the indirect method no integrals are required over the boundary; the boundary condition is enforced within the computational domain and the singularities individually satisfy the far-field condition. Thus, one may expect the effect due to the failure of satisfying the boundary condition outside the computational domain to be smaller than the effect due to the neglect of the far-field closure.

\subsection{Waves generated by a source-sink pair moving below a free surface}

Irrotational, incompressible flow is governed by the Laplace equation for the velocity potential $\phi$. On the free surface, the non-linear dynamic and kinematic boundary conditions are

$$
\begin{aligned}
\frac{\mathrm{D} \phi}{\mathrm{D} t} & =-z_{\mathrm{f}}+\frac{1}{2} \nabla \phi \cdot \nabla \phi, \\
\frac{\mathrm{D} \mathbf{x}_{\mathrm{f}}}{\mathrm{D} t} & =\nabla \phi,
\end{aligned}
$$

where $\mathbf{x}_{\mathrm{f}}=\left(x_{\mathrm{f}}, y_{\mathrm{f}}, z_{\mathrm{f}}\right)$ is the position vector of the fluid particle on the free surface and $\mathrm{D} / \mathrm{D} t$ is the substantial derivative following the fluid paticle. The $z$-axis is taken as positive upwards. Initially, there is no flow and the free surface is flat. The flow is generated by the motion of a source-sink pair that starts from rest. The speed of the disturbance pair is quickly brought to a steady value by a smooth function of time to avoid high-frequency content. The problem has been non-dimensionalized by taking the depth of the disturbance, $h$, and the gravitational acceleration $g$ to be unity. 
The free surface boundary conditions are integrated with respect to time to update the position of the fluid particles (the nodes) on the free surface and their velocity potential. The velocities of the fluid particles on the free surface at each time step are determined by the desingularized boundary integral method. The location of the singularities, $\mathbf{x}_{\mathbf{s}}$, are moved at each time step to a distance $L_{d}$ away from the nodes and normal to the surface (the average of the four normals at the nodal points). Material movement of the nodes is beneficial for this problem because they tend to naturally cluster near crests where curvature of the surface and velocity gradients are large. Since nodes cluster at these points, the desingularization distance decreases in a beneficial way as prescribed by (12). We use the indirect method with isolated sources because of its computational advantages and simplicity discussed in the previous sections. An additional advantage of the indirect method is that the velocities can be obtained directly once the strength of the singularity distribution is known, while the direct method requires numerical differentiation to obtain the tangential velocities.

The potential is expressed as a sum of (i) the source-sink disturbance pair at a distance $h$ below the undisturbed free surface, (ii) the image disturbance above the undisturbed free surface and (iii) a sum of $N$ sources of unknown strength in an array at a distance $L_{\mathrm{d}}$ above the disturbed free surface. The far-field condition is better satisfied when the images of the disturbance are used in the construction of the solution. The 'integral' equation for the unknowns $\sigma_{j}$ is

$$
\sum_{j=1}^{N} \frac{\sigma_{j}}{\left|\mathbf{x}_{\mathrm{f}}-\mathbf{x}_{\mathrm{s} j}\right|}=\phi\left(\mathbf{x}_{\mathrm{f}}\right)+\frac{\sigma(t)}{\left|\mathbf{x}_{\mathrm{f}}-\mathbf{x}_{\text {source }}\right|}-\frac{\sigma(t)}{\left|\mathbf{x}_{\mathrm{f}}-\mathbf{x}_{\text {sink }}\right|}-\frac{\sigma(t)}{\left|\mathbf{x}_{\mathrm{f}}-\mathbf{x}_{\text {source }}^{\prime}\right|}+\frac{\sigma(t)}{\left|\mathbf{x}_{\mathrm{f}}-\mathbf{x}_{\text {sink }}^{\prime}\right|},
$$

where $\phi\left(\mathbf{x}_{f}\right)$ is known and $\sigma(t)$ is the imposed strength of the disturbance source-sink pair. $\mathbf{x}_{\text {source }}$ and $\mathbf{x}_{\text {sink }}$ are the locations of the source and sink, and $\mathbf{x}_{\text {source }}^{\prime}$ and $\mathbf{x}_{\text {sink }}^{\prime}$ are the locations of their images. The source-sink pair disturbance (lying along a horizontal line) moves horizontally. The distance between the source and sink is chosen to be $0 \cdot 1$. The pair moves at speed $V(t)=\operatorname{Fr}\left(1-\mathrm{e}^{-4 t}\right)$ with the midpoint between the source and sink initially located at point $(5,0,-1)$. The Froude number $F r$ is defined by $V_{0} / \sqrt{ }(g h)$, where $V_{0}$ is the steady velocity of the disturbance. We choose $F r$ to be unity in this example.

After the $\sigma_{j}$ are determined, the velocities of the fluid particles on the free surface can be calculated. A fourth-order Runge-Kutta-Fehlberg method is used in the non-linear free surface integration. The method is first applied to waves generated by a sufficiently small disturbance such that linear wave theory is a good approximation. We compare the results of the present method using fully non-linear free surface conditions with a solution computed from a timedependent Green function for a Kelvin wave source that satisfies the linearized free surface conditions. ${ }^{16}$ Then we study non-linear free surface waves generated by a stronger disturbance.

Since the numerical accuracy is governed by the ratio between the element size and wavelength; we require the ratio between the element size in the $x$-direction and the wavelength to be less than $1 / 10$ to resolve the waves. The non-dimensional wavelength $\lambda$ is estimated by $\lambda=2 \pi F r^{2}$ using a two-dimensional linear theory. The initial free surface mesh grid extends from $0<x<20$ and $0<y<7.5$ (with a symmetry plane at $y=0$ ) and is initially divided into $40 \times 15$ elements. The nodes in the $x$-direction are equally spaced while the distance between two adjacent nodes in the increasing $y$-direction increases by $10 \%$. The nodal points serve as the material points. The time marching is conducted following the node points. The isolated sources are placed approximately perpendicular from the nodal points at a distance determined by (12), where the local mesh size $D_{\mathrm{m}}$ is the square root of the average area of four adjacent meshes, $l_{\mathrm{d}}$ is 1.0 and $\alpha$ is 0.5 . The magnitude of the disturbance for both the source and the sink is defined by $\sigma(t)=\sigma_{0}\left(1-\mathrm{e}^{-4 t}\right)$, where $\sigma_{0}=0.05$ for the linear case and $\sigma_{0}=0.75$ for the non-linear case. We use a time step of 0.2 in the time-marching procedure. 
Figure 13 shows the wave elevation along the symmetry plane $(y=0)$ at $t=10$ by the non-linear calculation and the linear calculation ${ }^{16}$ with the weaker disturbance. The time convolution integrals in the linear calculation are obtained numerically. As seen, the non-linear and linear results agree very well. Independent computations using (a) a smaller computational domain (with the same mesh spacing within $0<x<15$ and $0<y<7.5$ ), (b) finer mesh grids $(80 \times 15$ and $40 \times 30$ with the same computational domain) and (c) doubling of the time increment result in negligible difference for the non-linear calculation. This indicates that even for the small-disturbance example studied here, the small differences in Figure 13 are primarily due to non-linear effects.

Figure 14 shows the elevation along the symmetry plane at $t=10$ for the non-linear case. The difference between the non-linear and linear results are due to the non-linear effect of the free surface conditions. The figure indicates that non-linear effects are stronger near the second crest. A three-dimensional view of the non-linear waves at $t=14$ is given in Figure 15 with $40 \times 15$ elements on the computational domain, $0<x<20$ and $0<y<7.5$. This truncation requires 10.5 CPU seconds to form the influence matrix and 48.5 s for GMRES to solve the system of equations (less for small $t$ ). Since the Runge-Kutta procedure requires four evaluations of the integral equation per time step, the computation shown in Figure 15 requires approximately $20 \mathrm{~min}$ on the Apollo DN 10000.

To study artificial effects (such as wave reflection) caused by far-field truncation on longer-time simulations, the weak disturbance was simulated on the two differently sized computational domains mentioned above with identical mesh spacing. Figure 16 shows the results using the two domains. Small differences are seen in the two computations before the wave front hits the edge of the smaller domain. Moreover, even after the wave front passes this edge, significant differences

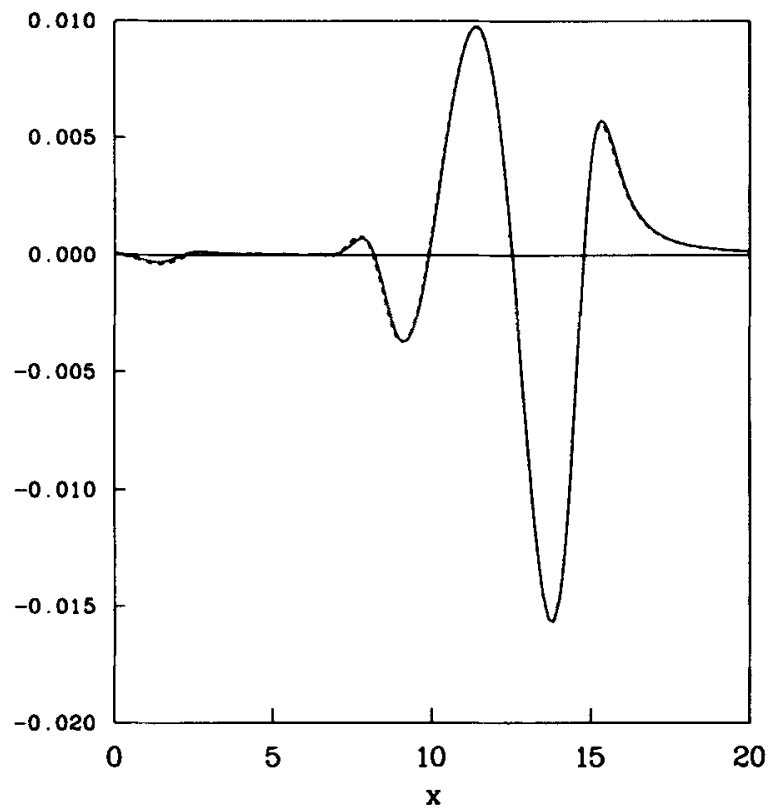

Figure 13. Free surface elevation along symmetry plane at $t=10\left(\right.$ linear case: $\left.\sigma_{0}=0.05, F r=1\right)$, non-linear result; ,--- linear result 


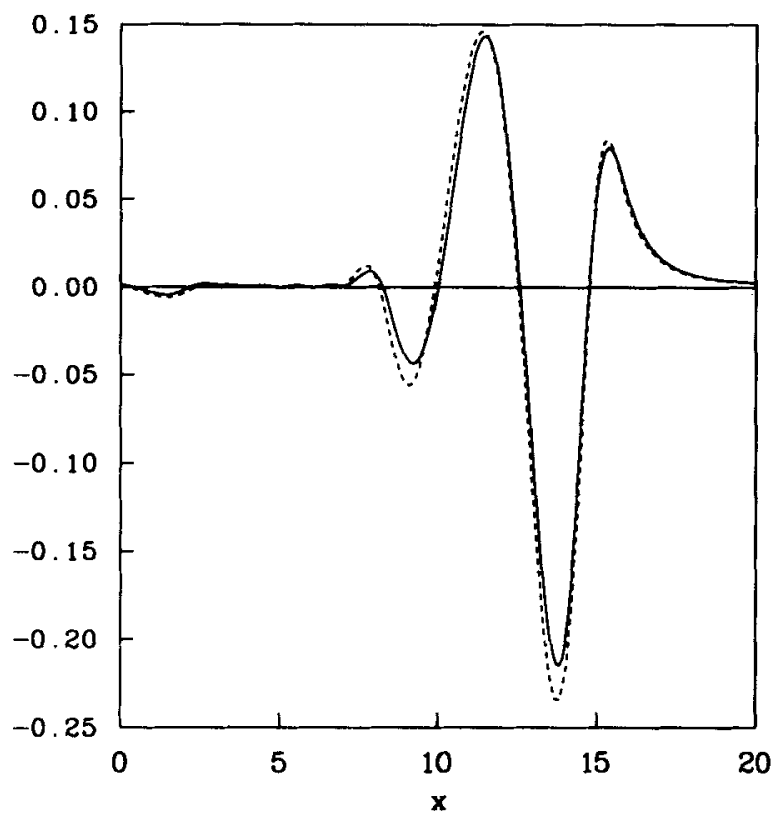

Figure 14. Free surface elevation along symmetry plane at $t=10$ (non-linear case: $\left.\sigma_{0}=0.75, F r=1\right)$; - , non-linear result, ----, linear result

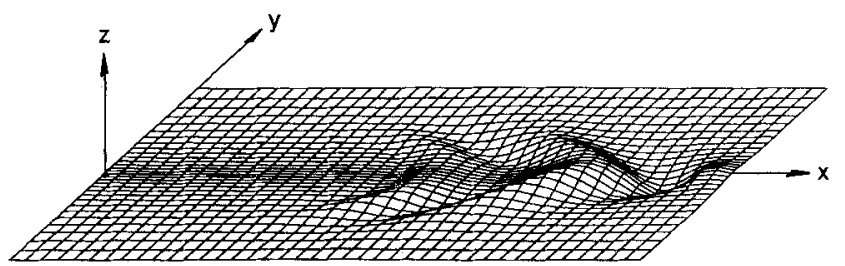

Figure 15. Three-dimensional view of non-linear wave at $t=14\left(\sigma_{0}=0.75, F r=1\right)$

are not apparent until the first crest passes the edge. After that, the error starts to propagate into the entire domain. This is remarkable, especially considering that the edges of the computational domain require no special treatment such as one-sided derivatives, not to mention a radiationtype boundary condition. This is due to the fact that the indirect method using material nodal points does not require any spatial derivatives when the velocities are obtained directly from the singularities. Our attempts to solve the identical problem using the direct method (not shown) had extraneous reflections almost immediately at the truncated boundary where a boundary condition $(\phi=0)$ was imposed. It appears that the indirect method allows the use of a considerably smaller computational domain.

\section{CONCLUSIONS}

The non-singular formulation significantly reduces the time required to compute the matrix of influence functions. The resulting systems of linear equations are still adequately well conditioned 


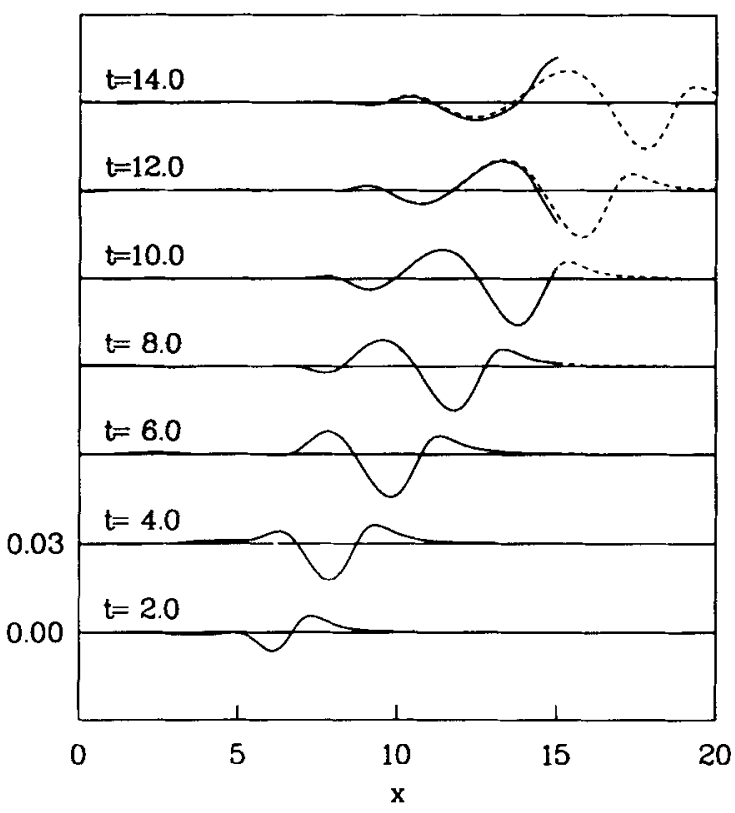

Figure 16. Effect of computational domain size on wave elevation along symmetry plane $\left(\sigma_{0}=0.05, F r=1\right)$ : , small domain $(0<x<15) ;---$, large domain $(0<x<20)$

to allow efficient iterative solutions. Accurate solutions can be obtained by the desingularized boundary method for a large range of desingularization distances of the order of the mesh size $\left(l_{\mathrm{d}} \approx 1\right)$. For our examples we find that the indirect method is more efficient than the direct method. It is easy to code and requires less computational effort. In addition, the indirect method performs better in problems with boundaries extending to infinity. Non-linear wave calculations using a time-marching procedure were greatly facilitated using the desingularized boundary integral method. Only small numerical reflections at the computational boundaries were observed.

\section{ACKNOWLEDGEMENT}

This work was supported under the Program in Ship Hydrodynamics at The University of Michigan, funded by The University Research Initiative of the Office of Naval Research, Contract Number N000184-86-K-0684.

\section{REFERENCES}

1. M. S. Longuet-Higgins and C. D. Cokelet, 'The deformation of steep surface waves on water: I. A numerical method of computation', Proc. R. Soc. Lond. A, 350, 1-26 (1976).

2. G. R. Baker, D. I. Meiron and S. A. Orszag, 'Generalized vortex methods for free surface flow problems', J. Fluid Mech., 123, 477-501 (1982).

3. D. G. Dommermuth and D. K. P. Yue, 'Numerical simulations of nonlinear axisymmetric flows with a free surface', J. Fluid Mech., 178, 195-219 (1987).

4. T. von Kármán, 'Calculation of pressure distribution on airship hulls', NACA Technical Memorandum No. $574,1930$.

5. V. Kupradze, 'On the approximate solution of problems in mathematical physics', Russ. Math. Surveys, 22, 59-107 (1967). 
6. U. Heise, 'Numerical properties of integral equations in which the given boundary values and the solutions are defined on different curves', Comput. Struct., 8, 199-205 (1978).

7. P. A. Martin, 'On the null-field equations for water-wave radiation problems', J. Fluid Mech., 113, 315-332 (1981).

8. P. S. Han and M. D. Olson, 'An adaptive boundary element method', Int.j. numer. methods eng., 24, 1187-1202 (1987).

9. R. L. Johnston and G. Fairweather, 'The method of fundamental solutions for problems in potential flow', Appl. Math. Modeling, 8, 265-270 (1984).

10. W. C. Webster, 'The flow about arbitrary, three-dimensional smooth bodies', J. Ship Res. 19, 206-218 (1975).

11. W. W. Schultz and S. W. Hong, 'Solution of potential problems using an overdetermined complex boundary integral method', J. Comput. Phys., 84, 414-440 (1989).

12. G. Jensen, Z. X. Mi and H. Söding, 'Rankine source methods for numerical solutions of the steady wave resistance problem', 16th Symp. on Naval Hydrodynamics, University of California, Berkeley, USA, 1986, pp. 575-582.

13. C. A. Brebbia and R. Butterfield, 'Formal equivalence of direct and indirect boundary mothods', Appl. Math. Modeling, 2, 132-134 (1978).

14. Y. Saad and M. H. Schultz, 'GMRES: a generalized minimal residual algorithm for solving nonsymmetric linear systems', SIAM J. Sci. Stat. Comput., 7, 856-869 (1986).

15. J. N. Newman, 'Distributions of sources and normal dipoles over a quadrilateral panel', J. Eng. Math., 20, 113-126 (1986).

16. B. King, 'Time domain analysis of wave exciting forces on ships and bodies', Ph.D. Thesis, Department of Naval Architecture and Marine Engineering, University of Michigan, Ann Arbor, USA, 1987. 